\title{
A Convex Programming-based Algorithm for Mean Payoff Stochastic Games with Perfect Information*
}

\author{
Endre Boros ${ }^{\dagger} \quad$ Khaled Elbassioni $^{\ddagger} \quad$ Vladimir Gurvich $^{\S} \quad$ Kazuhisa Makino
}

\begin{abstract}
We consider two-person zero-sum stochastic mean payoff games with perfect information, or BWR-games, given by a digraph $G=(V, E)$, with local rewards $r: E \rightarrow \mathbb{Z}$, and three types of positions: black $V_{B}$, white $V_{W}$, and random $V_{R}$ forming a partition of $V$. It is a long-standing open question whether a polynomial time algorithm for BWR-games exists, even when $\left|V_{R}\right|=0$. In fact, a pseudo-polynomial algorithm for BWR-games would already imply their polynomial solvability. In this short note, we show that BWR-games can be solved via convex programming in pseudo-polynomial time if the number of random positions is a constant.
\end{abstract}

\section{Introduction}

We consider two-person zero-sum stochastic games with perfect information and mean payoff: Let $G=(V, E)$ be a digraph whose vertex-set $V$ is partitioned into three subsets $V=V_{B} \cup V_{W} \cup V_{R}$ that correspond to black, white, and random positions, controlled respectively, by two players, MIN - the minimizer and MAX - the maximizer, and by nature. We also fix a local reward function $r: E \rightarrow \mathbb{Z}$, and probabilities $p(v, u)>0$ for all arcs $(v, u)$ going out of $v \in V_{R}$. We assume that $\sum_{u \mid(v, u) \in E} p(v, u)=1$, for all $v \in V_{R}$. Vertices $v \in V$ and arcs $e \in E$ are called positions and moves, respectively. The game begins at time $t=0$ in the initial position $s_{0}=v_{0}$. In a general step, in time $t$, we are at position $s_{t} \in V$. The player who controls $s_{t}$ chooses an outgoing arc $e_{t+1}=\left(s_{t}, v\right) \in E$, and the game moves to position $s_{t+1}=v$. If $s_{t} \in V_{R}$ then an outgoing arc is choses with the given probability $p\left(s_{t}, s_{t+1}\right)$. We assume that every vertex in $G$ has an outgoing arc. In general, the strategy of the player is a policy by which (s)he chooses the outgoing arcs from the vertices (s)he controls. This policy may involve the knowledge of the previous steps as well as probabilistic decisions. We call a strategy stationary if it does not depend on the history and pure if it does not involve probabilistic decisions. For this type of games, it will be enough to consider only such strategies, since these games are known to be (polynomially) equivalent [BEGM13a] to the perfect information stochastic games considered by Gillette Gil57, LL69.

In the course of this game players and nature generate an infinite sequence of edges $\mathbf{p}=$ $\left(e_{1}, e_{2}, \ldots\right)$ (a play) and the corresponding real sequence $r(\mathbf{p})=\left(r\left(e_{1}\right), r\left(e_{2}\right), \ldots\right)$ of local rewards. There is a global payoff function $\phi$ that maps any local reward sequence to a real number, and it is assumed that Min pays MAX the amount $\phi(r(\mathbf{p}))$ resulting from the play. Naturally, MAX's aim is to create a play which maximizes $\phi(r(\mathbf{p}))$, while Min tries to minimize it. (Let us note that the local reward function $r: E \rightarrow \mathbb{R}$ may have negative values, and $\phi(r(\mathbf{p}))$ may also be negative, in

\footnotetext{
*Part of this research was done at the Mathematisches Forschungsinstitut Oberwolfach during a stay within the "Research in Pairs" Program from July 26, 2015-August 15, 2015. The research of the third author has been partially funded by the Russian Academic Excellence Project '5-100'. The fourth author was partially supported by KAKENHI Grant Numbers 24106002 and 26280001.

${ }^{\dagger}$ MSIS Department and RUTCOR, Rutgers University, 100 Rockafellar Road, Livingston Campus Piscataway, NJ 08854, USA; (Endre.Boros@rutgers.edu)

${ }_{\ddagger}$ Masdar Institute of Science and Technology, P.O.Box 54224, Abu Dhabi, UAE; (kelbassioni@masdar.ac.ae)

$\S$ MSIS Department and RUTCOR, Rutgers University, 100 Rockafellar Road, Livingston Campus Piscataway, NJ 08854, USA; and National Research University, Higher School of Economics, Moscow, Russia; (Vladimir.Gurvich@gmail.com)

IResearch Institute for Mathematical Sciences (RIMS) Kyoto University, Kyoto 606-8502, Japan; (makino@kurims.kyoto-u.ac.jp)
} 
which case Max has to pay Min $-\phi(r(\mathbf{p}))$. Let us also note that $r(\mathbf{p})$ is a random variable since random transitions occur at positions in $V_{R}$.) Here $\phi$ stands for the limiting mean payoff

$$
\phi(r(\mathbf{p}))=\liminf _{T \rightarrow \infty} \frac{\sum_{i=1}^{T} \mathbb{E}\left[r\left(e_{i}\right)\right]}{T},
$$

where $\mathbb{E}\left[r\left(e_{i}\right)\right]$ is the expected reward incurred at step $i$ of the play.

As usual, a pair of (not necessarily pure or stationary) strategies is a saddle point (or equilibrium) if neither of the players can improve individually by changing her/his strategy. The corresponding $\phi(r(\mathbf{p}))$ is the value $\mu_{\mathcal{G}}\left(v_{0}\right)$ of the game with respect to initial position $v_{0}$. Such a pair of strategies are called optimal; furthermore, it is called uniformly optimal if it provides the value of the game for any initial position. It is known Gil57, LL69 that every such game has a pair of uniformly optimal pure stationary strategies. A BWR-game is said to be ergodic if $\mu_{\mathcal{G}}(v)=\mu$ for all $v \in V$, that is, the value is the same from each initial position.

This class of $B W R$-games was introduced in GKK88; see also [CH08. The special case when $V_{R}=\emptyset, B W$-games, is also known as cyclic games. They were introduced for the complete bipartite digraphs in Mou76b, Mou76a, for all (not necessarily complete) bipartite digraphs in [EM79], and for arbitrary digraph 1 in GKK88. A more special case was considered extensively in the literature under the name of parity games [BV01a, BV01b, CJH04, Hal07, Jur98, JPZ06], and later generalized also to include random positions in CH08. A BWR-game is reduced to a minimum mean cycle problem in case $V_{W}=V_{R}=\emptyset$, see, e.g., Kar78. If one of the sets $V_{B}$ or $V_{W}$ is empty, we obtain a Markov decision process (MDP), which can be expressed as a linear program; see, e.g., MO70. Finally, if both are empty, $V_{B}=V_{W}=\emptyset$, we get a weighted Markov chain. For BW-games several pseudo-polynomial and subexponential algorithms are known GKK88, KL93, ZP96, Pis99, BV01a, BV01b, HBV04, BV05, BV07, Hal07, Sch09, Vor08; ; see also [JPZ06 for parity games. Besides their many applications (see e.g. [Lit96, Jur00]), all these games are of interest to Complexity Theory: It is known [KL93, ZP96] that the decision problem "whether the value of a BW-game is positive" is in the intersection of NP and co-NP. Yet, no polynomial algorithm is known for these games, see e.g., the survey by Vorobyov Vor08. A similar complexity claim can be shown to hold for BWR-games, see AM09, BEGM13a.

\section{Main result}

The computational complexity of stochastic games with perfect information is an outstanding open question; see, e.g., the survey [RF91]. While there are numerous pseudo-polynomial algorithms known for the BW-case, it is a challenging open question whether a pseudo-polynomial algorithm exists for BWR-games, as the existence of such an algorithm would imply also the polynomial solvability of this class of games AM09.

In BEGM13b, BEGM15, we gave a pseudo-polynomial algorithm for BWR-games when the number of random positions is fixed. In this note we show that one can obtain a similar result via convex programming, combined with some of the ideas in BEGM13b, BEGM15.

For a BWR-game $\mathcal{G}$ let us denote by $n=\left|V_{W}\right|+\left|V_{B}\right|+\left|V_{R}\right|$ the number of positions, by $k=\left|V_{R}\right|$ the number of random positions, and assume (without loss of generality) that all local rewards are non-negative integers with maximum value $U$ and all transition probabilities are rational with common denominator $D$. The main result of this paper is as follows.

Theorem 1 A BWR-game $\mathcal{G}$ can be solved in poly $\left(n, U, D^{k}\right)$ time via convex programming.

This theorem extends the result by Schewe [Sch09], where it was shown that solving BW-games can be reduced to solving linear programming problems with pseudo-polynomial bit length.

According to the results in BEGM13b, BEGM15, to get a pseudo-polynomial algorithm for BWR-games, it is enough to have pseudo-polynomial routines for: (i) solving BW-games; (ii) solving

\footnotetext{
${ }^{1}$ In fact, BW-games on arbitrary digraphs can be polynomially reduced to BW-games on bipartite digraphs BEGM13a]; moreover, the latter class can further be reduced to BW-games on complete bipartite digraphs [CHKN14].
} 
ergodic BWR-games; and (iii) finding the top and bottom classes in a non-ergodic BWR-game (that is, the sets of positions with highest and lowest values).

There are several pseudo-polynomial algorithms for solving BW-games, e.g., GKK88, Pis99, ZP96]. One may also use the LP-based algorithm given in [Sch09]. For (ii) we show in Section [5how to obtain the top (resp., bottom) class in a BWR-game, and a pair of strategies solving the game induced by the top (resp., bottom) class. This also provides an algorithm for ergodic BWR-games as required in (iii).

\section{Potential transformations and canonical forms}

Given a BWR-game $\mathcal{G}=(G, p, r)$, let us introduce a mapping $x: V \rightarrow \mathbb{R}$, whose values $x(v)$ will be called potentials, and define the transformed reward function $r_{x}: E \rightarrow \mathbb{R}$ as:

$$
r_{x}(v, u)=r(v, u)+x(v)-x(u), \text { where }(v, u) \in E .
$$

It is not difficult to verify that the obtained game $\mathcal{G}^{x}$ and the original game $\mathcal{G}$ are equivalent (see [BEGM13a]). In particular, their optimal (pure stationary) strategies coincide, and their value functions also coincide: $\mu_{\mathcal{G}^{x}}=\mu_{\mathcal{G}}$.

It is known that for BW-games there exists a potential transformation such that, in the obtained game the locally optimal strategies are globally optimal, and hence, the value and optimal strategies become obvious GKK88. This result was extended for the more general class of BWR-games in [BEGM13a]: in the transformed game, the equilibrium value $\mu_{\mathcal{G}}(v)=\mu_{\mathcal{G}^{x}}(v)$ is given simply by the maximum local reward for $v \in V_{W}$, the minimum local reward for $v \in V_{B}$, and the average local reward for $v \in V_{R}$. In this case we say that the transformed game is in canonical form. To define this more formally, let us use the following notation throughout this section: Given functions $f: E \rightarrow \mathbb{R}$ and $g: V \rightarrow \mathbb{R}$, we define the functions $M[f], M[g]: V \rightarrow \mathbb{R}$.

$$
\begin{gathered}
M[f](v)= \begin{cases}\max _{u \mid(v, u) \in E} f(v, u), & \text { for } v \in V_{W}, \\
\min _{u \mid(v, u) \in E} f(v, u), & \text { for } v \in V_{B}, \\
\sum_{u \mid(v, u) \in E} p(v, u) f(v, u), & \text { for } v \in V_{R} .\end{cases} \\
M[g](v)= \begin{cases}\max _{u \mid(v, u) \in E} g(u), & \text { for } v \in V_{W}, \\
\min _{u \mid(v, u) \in E} g(u), & \text { for } v \in V_{B}, \\
\sum_{u \mid(v, u) \in E} p(v, u) g(u), & \text { for } v \in V_{R} .\end{cases}
\end{gathered}
$$

We say that a BWR-game $\mathcal{G}$ is in (strong) canonical form if there exist vectors $\mu, x \in \mathbb{R}^{V}$ such that

(C1) $\mu=M[\mu]=M\left[r_{x}\right]$ and,

(C2) for every $v \in V_{W} \cup V_{B}$, every move $(v, u) \in E$ such that $\mu(v)=r_{x}(v, u)$ must also have $\mu(v)=\mu(u)$, or in other words, every locally optimal move $(v, u)$ is globally optimal.

Theorem 2 ([BEGM13a]) For each $B W R$-game $\mathcal{G}$ there is a potential transformation $x \in \mathbb{R}^{V}$ that brings $\mathcal{G}$ to canonical form with $\|x\|_{\infty} \leq L:=n U k(2 D)^{k}$. Furthermore, in a game in canonical form we have $\mu_{\mathcal{G}}=M\left[r_{x}\right]$.

In this paper, we will provide a convex programming formulation based on the existence of potential transformations.

We will need the following upper bound on the required accuracy.

Lemma 1 ([BEGM10, BEGM15]) For any position $v$ in the top (resp., bottom) class in a BWRgame $\mathcal{G}$, the value $\mu_{\mathcal{G}}(v)$ is a rational number with a denominator at most $\sqrt{k} 2^{k / 2} D^{k+1}$.

Lemma 2 Consider a BWR-game $\mathcal{G}$ and denote by 1 the vector of all ones. Then there exists a potential vector $x \in \mathbb{R}^{V}$ and $t \in \mathbb{R}$ such that $M\left[r_{x}\right] \geq t \mathbf{1}$ if and only if $\mu_{\mathcal{G}} \geq t \mathbf{1}$. 
Proof Indeed, if MAX (Min) applies a locally optimal strategy $s_{W}$ in the transformed game $\mathcal{G}^{x}$ then after every own move (s)he will get (pay) at least $t$, while for each move of the opponent the local reward will be at least (at most) $t$, and finally, for each random position the expected local reward is at least $t$. Thus, the expected local reward $\mathbb{E}\left[r_{x}\left(e_{i}\right)\right]$ at each step of the play is at least $t$. Hence, by (1), strategy $s_{W}$ guarantees MAX at least $t$ from any starting position.

The other direction follows from Theorem 2 .

A symmetric version of Lemma 2 can also be obtained by similar arguments.

Lemma 3 Consider a BWR-game $\mathcal{G}$. Then there exists a potential vector $x \in \mathbb{R}^{V}$ and $t \in \mathbb{R}$ such that $M\left[r_{x}\right] \leq t \mathbf{1}$ if and only if $\mu_{\mathcal{G}} \leq t \mathbf{1}$.

\section{The convex programs}

The following simple facts relate the softmax (resp., softmin) to the maximimum (resp., minimum) of a set of numbers.

Fact 1 For any numbers $a_{1}, \ldots, a_{n} \in \mathbb{R}$ and $b>1$ :

(i) $\max _{i} a_{i} \leq \log _{b} \sum_{i} b^{a_{i}} \leq \max _{i} a_{i}+\log _{b} n$;

(ii) $\min _{i} a_{i} \geq-\log _{b} \sum_{i} b^{-a_{i}} \geq \min _{i} a_{i}-\log _{b} n$.

Proof This follows from the fact the trivial inequalities $b^{\max _{i} a_{i}} \leq \sum_{i} b^{a_{i}} \leq n b^{\max _{i} a_{i}}$.

Fact 2 Let $\alpha_{1}, \ldots, \alpha_{n}>0$ be given numbers such that $\sum_{i=1}^{n} \alpha_{i}=1$. Then the function $f(x)=$ $\prod_{i=1}^{n} x_{i}^{\alpha_{i}}$ is concave for $x \geq 0$.

Proof Note that for any $x, y \in \mathbb{R}_{+}^{n}$, if for some $i, x_{i}=0$ then for any $\lambda \in[0,1]$,

$$
\begin{aligned}
\lambda f(x)+(1-\lambda) f(y) & =(1-\lambda) f(y) \\
& =(1-\lambda) \prod_{i=1}^{n} y_{i}^{\alpha_{i}}=\prod_{i=1}^{n}\left((1-\lambda) y_{i}\right)^{\alpha_{i}} \\
& \leq \prod_{i=1}^{n}\left(\lambda_{i} x_{i}+(1-\lambda) y_{i}\right)^{\alpha_{i}}=f(\lambda \mathbf{x}+(1-\lambda) y) .
\end{aligned}
$$

Thus, it is enough to show that $\nabla^{2} f(x)$ is a negative semi-definite matrix for $\mathbf{x}>0$. Note that

$$
\begin{aligned}
\frac{\partial f}{\partial x_{i}} & =\frac{\alpha_{i}}{x_{i}} f(x), \text { for } i=1, \ldots, n \\
\frac{\partial^{2} f}{\partial x_{i}^{2}} & =\frac{\alpha_{i}\left(\alpha_{i}-1\right)}{x_{i}^{2}} f(x), \text { for } i=1, \ldots, n \\
\frac{\partial^{2} f}{\partial x_{i} \partial x_{j}} & =\frac{\alpha_{i} \alpha_{j}}{x_{i} x_{j}} f(x), \text { for } i, j=1, \ldots, n, i \neq j .
\end{aligned}
$$

Consider any $y \in \mathbb{R}^{n}$. Then

$$
\begin{aligned}
y^{T} \nabla^{2} f(x) y & =\left(\sum_{i} \alpha_{i}\left(\alpha_{i}-1\right) \frac{y_{i}^{2}}{x_{i}^{2}}+\sum_{i \neq j} \alpha_{i} \alpha_{j} \frac{y_{i} y_{j}}{x_{i} x_{j}}\right) f(x) \\
& =\left(\left(\sum_{i} \alpha_{i} \frac{y_{i}}{x_{i}}\right)^{2}-\sum_{i} \alpha_{i}\left(\frac{y_{i}}{x_{i}}\right)^{2}\right) f(x) \leq 0,
\end{aligned}
$$

where the last inequality follows from Jensen's inequality applied to the convex function $f(w)=w^{2}$. 
Given $t \in \mathbb{R}$, let us replace the max operator in the system $M\left[r_{x}\right] \geq t$ by the softmax approximation:

$$
\begin{array}{rrr}
\log _{b} \sum_{u \mid(v, u) \in E} b^{r(v, u)+x(v)-x(u)} \geq t, & \text { for } v \in V_{W}, \\
r(v, u)+x(v)-x(u) \geq t, & \text { for } u \text { s.t. }(v, u) \in E & \text { for } v \in V_{B}, \\
\sum_{u \mid(v, u) \in E} p(v, u)(r(v, u)+x(v)-x(u)) & \geq t, & \text { for } v \in V_{R},
\end{array}
$$

where the constant $b$ will be determined later. Defining the new variables $y(v):=b^{-x(v)}$, we can rewrite (3)-(5) as follows:

$$
\begin{array}{rlrl}
\sum_{u \mid(v, u) \in E} b^{r(v, u)} y(u) & \geq b^{t} y(v), & \text { for } v \in V_{W}, \\
b^{r(v, u)} y(u) \geq b^{t} y(v), & \text { for } u \text { s.t. }(v, u) \in E & \text { for } v \in V_{B}, \\
\prod_{u \mid(v, u) \in E}\left(b^{r(v, u)} y(u)\right)^{p(v, u)} \geq b^{t} y(v), & \text { for } v \in V_{R} .
\end{array}
$$

Note that $y(v)>0$ if and only if $x(v)$ is finite. In fact, since we may assume by Theorem 2 that $\|x\|_{\infty} \leq L$, we may add also the inequalities:

$$
b^{-L} \leq y(v) \leq b^{L}, \quad \text { for } v \in V .
$$

Note that, without the lower bounds $y(v) \geq b^{-L}$, the system (6)-(8) is always feasible. As we shall see later, it will be necessary to test the feasibility of the system with $y(v)>0$ for some $v \in V$. For convenience, let us write more generally the following set of upper and lower bounds, where $V^{\prime} \subseteq V$ is to be chosen later:

$$
0 \leq y(v) \leq b^{L}, \quad \text { for } v \in V, \text { and } y(v) \geq b^{-L}, \quad \text { for } v \in V^{\prime} .
$$

Similarly, we replace the min operator in the system $M\left[r_{x}\right] \leq t$ by the softmin approximation:

$$
\begin{array}{rlrl}
r(v, u)+x(v)-x(u) & \leq t, & \text { for } u \text { s.t. }(v, u) \in E & \text { for } v \in V_{W}, \\
-\log _{b} \sum_{u \mid(v, u) \in E} b^{-r(v, u)-x(v)+x(u)} \leq t, & \text { for } v \in V_{B}, \\
\sum_{u \mid(v, u) \in E} p(v, u)(r(v, u)+x(v)-x(u)) & \leq t, & \text { for } v \in V_{R},
\end{array}
$$

and defining the new variables $y(v):=b^{x(v)}$, we can rewrite (10)-(12) as follows:

$$
\begin{array}{rlrl}
b^{-r(v, u)} y(u) & \geq b^{-t} y(v), & \text { for } u \text { s.t. }(v, u) \in E & \text { for } v \in V_{W}, \\
\sum_{u \mid(v, u) \in E} b^{-r(v, u)} y(u) & \geq b^{-t} y(v), & & \text { for } v \in V_{B}, \\
\prod_{u \mid(v, u) \in E}\left(b^{-r(v, u)} y(u)\right)^{p(v, u)} \geq b^{-t} y(v), & \text { for } v \in V_{R},
\end{array}
$$

together with the lower and upper bounds:

$$
0 \leq y(v) \leq b^{L}, \quad \text { for } v \in V, \text { and } y(v) \geq b^{-L}, \quad \text { for } v \in V^{\prime} .
$$




\section{Solving the convex programs}

We will use the ellipsoid method Kha80, Kha84, GLS88. For this we need to show that the separation problem can be solved in polynomial time. For convenience, let us consider the following relaxation of the convex programs (6)-(9) and (13)-(16):

$$
\begin{array}{cr}
\sum_{u \mid(v, u) \in E} b^{r(v, u)} y(u) \geq b^{t} y(v)-\delta, & \text { for } v \in V_{W}, \\
b^{r(v, u)} y(u) \geq b^{t} y(v)-\delta, & \text { for } u \text { s.t. }(v, u) \in E \text { for } v \in V_{B}, \\
\prod_{u \mid(v, u) \in E}\left(b^{r(v, u)} y(u)\right)^{p(v, u)} \geq b^{t} y(v)-\delta, & \text { for } v \in V_{R} . \\
0 \leq y(v) \leq b^{L}+\delta, \quad \text { for } v \in V, \text { and } b^{L} y(v) \geq 1, & \text { for } v \in V^{\prime} . \\
\sum_{u \mid(v, u) \in E} b^{-r(v, u)} y(u) \geq b^{-t} y(v)-\delta, & \text { for } v \in V_{W}, \\
\prod^{-r(v, u)} y(u) \geq b^{-t} y(v)-\delta, & \text { for } u \text { s.t. } \quad(v, u) \in E \text { for } v \in V_{B}, \\
\left(b^{-r(v, u)} y(u)\right)^{p(v, u)} \geq b^{-t} y(v)-\delta, & \text { for } v \in V_{R} .
\end{array}
$$

where $\delta>0$ is a rational number that will be chosen appropriately. Let $K$ and $K_{\delta}$ be the set of $y \in \mathbb{R}^{E}$ satisfying (60)-(91) and (17)-(20), respectively. Similarly, Let $K^{\prime}$ and $K_{\delta}^{\prime}$ be the set of $y \in \mathbb{R}^{E}$ satisfying (13)-(16) and (21)-(24), respectively.

In our application, we will set $b=n^{4 \Lambda}$ and $t \in[0, U]$ to be a rational number with denominator $\Lambda:=\sqrt{k} 2^{k / 2} D^{k+1}$. In particular, $b^{ \pm t}$ is a rational number of bit length $\left\langle b^{ \pm t}\right\rangle=O(\Lambda \log n)$. Also by assuming without loss of generality (by scaling $r$ and replacing $U$ by $U D$ ) that $r(v, u)$ is a multiple of $D, b^{ \pm r(v, u)}$ is a rational number of bit length $\left\langle b^{ \pm r(v, u)}\right\rangle=O(\Lambda U D \log n)$.

Claim 1 For $0<\epsilon \leq b^{-t} \delta$ and any $y \in K$ (resp., $y \in K^{\prime}$ ), the box $\left\{y^{\prime} \in \mathbb{R}^{n} \mid y \leq y^{\prime} \leq y+\epsilon \cdot \mathbf{1}\right\}$ is contained in $K_{\delta}$ (resp., $K_{\delta}^{\prime}$ ), where $\mathbf{1}$ is the $n$-dimensional vector of all ones. In particular, if $K \neq \emptyset$ (resp., $K^{\prime} \neq \emptyset$ ) then $K_{\delta}$ (resp., $K_{\delta}^{\prime}$ ) is full-dimensional.

Proof We prove the statement for $K_{\delta}$; the proof for $K_{\delta}^{\prime}$ is similar. Clearly, $K \subseteq K_{\delta}$ for $\delta \geq 0$. Furthermore, for any $y \in K$ and $S \subseteq V$, the vector $y^{\prime}$ obtained from $y$ by setting $y^{\prime}(u):=y(u)+\epsilon$ for $u \in S$ and $y^{\prime}(u):=y(u)$ for $u \in V \backslash S$ satisfies (6)-(9). Indeed, the left-hand sides of (17)-(19) increase when $y$ is increased in the components corresponding to $S$, while the right-hand sides are at most $b^{t} y(v)+b^{t} \epsilon-\delta \leq b^{t} y(v)$. Also by (9), $y^{\prime}(v) \leq y(v)+\epsilon \leq b^{L}+\epsilon \leq b^{L}+\delta$, so $y^{\prime}$ satisfies (17)-(20).

Now we consider the (semi-weak) separation problem for $K_{\delta}$ (resp., $K_{\delta}^{\prime}$ ):

Given $\bar{y} \in \mathbb{Q}^{n}$ and $0<\delta^{\prime} \in \mathbb{Q}$, either assert that $\bar{y} \in K_{\delta}$ (resp., $\bar{y} \in K_{\delta}^{\prime}$ ) or find a vector $c \in \mathbb{Q}^{n}$ such that $c^{T} y+\delta^{\prime} \geq c^{T} \bar{y}$ for all $y \in K_{\delta}$ (resp., $y \in K_{\delta}^{\prime}$ ).

Claim 2 The separation problems for $K_{\delta}$ and $K_{\delta}^{\prime}$ can be solved in $\operatorname{poly}\left(U, D, \Lambda, \log n,\langle\bar{y}\rangle,\left\langle\delta^{\prime}\right\rangle\right)$ time.

Proof We present the proof for $K_{\delta}$; the proof for $K_{\delta}^{\prime}$ is similar. Clearly, we can check in poly $\left(\langle\bar{y}\rangle,\left\langle b^{t}\right\rangle,\langle\delta\rangle, n\right)$ time if $\bar{y}$ satisfies the linear inequalities (17), (18) and (20); if one is violated, the corresponding hyperplane defines a (exact) separator $c \in \mathbb{Q}^{n}$ and we are done. Assume therefore that $\bar{y}$ satisfies (17), (18) and (20). Let us now consider an inequality of the form (19) corresponding 
to $v \in V$ violated by $\bar{y}$. Let $f(y)=\prod_{u \mid(v, u) \in E}\left(b^{r(v, u)} y(u)\right)^{p(v, u)}-b^{t} y(v):=A \cdot g(y)-B \cdot y(v)$, where $A:=\prod_{u \mid(v, u) \in E} b^{r(v, u) p(v, u)}, B:=b^{t}$ and $g(y):=\prod_{u \mid(v, u) \in E} y(u)^{p(v, u)}$. Note that $f(\bar{y})<-\delta$ if and only if $A^{D} \cdot g(\bar{y})^{D}<(B \cdot \bar{y}(v)-\delta)^{D}$, which can be checked in poly $(\langle\bar{y}\rangle,\langle\delta\rangle, n, \log D)$ time, as both $A^{D}$ and $B^{D}$ are non-negative integer 2. Without loss of generality 3 , we assume that for every $u \in V$ there is exactly one edge $(v, u) \in E$. Then $\nabla f(y):=A \cdot\left(\frac{p(v, u)}{y(u)}: u \in V\right) g(y)-B \mathbf{1}_{v}$, where $\mathbf{1}_{v}$ is the unit dimensional vector with 1 in position $v$. Then the inequality $-\delta \leq f(y) \leq$ $f(\bar{y})+\nabla f(\bar{y})^{T}(y-\bar{y})<-\delta+\nabla f(\bar{y})^{T}(y-\bar{y})$, valid for all $y \in K_{\delta}$ by concavity of $f(y)$, gives a separating inequality:

$$
\nabla f(\bar{y})^{T} y>\nabla f(\bar{y})^{T} \bar{y} \quad \text { for all } y \in K_{\delta} .
$$

Note that the vector $\nabla f(\bar{y})$ can be irrational (it is irrational whenever $g(\bar{y})$ is). We define a rational approximation $\tilde{g}$ such that $\tilde{g} \geq g(\bar{y}) \geq \tilde{g}-\frac{\delta^{\prime}}{A}$ and $c:=A \cdot\left(\frac{p(v, u)}{\bar{y}(u)}: u \in V\right) \tilde{g}-B \mathbf{1}_{v}$. Since $r(v, u)$ is assumed to be integer multiple of $D, A$ is an integer and hence $\tilde{g}$ is a rational number of bit length $\langle\tilde{g}\rangle=\langle A\rangle+\left\langle\delta^{\prime}\right\rangle$. It follows also that $c$ is a rational vector of bit length $\operatorname{poly}\left(U D \Lambda \log n,\langle\bar{y}\rangle,\left\langle\delta^{\prime}\right\rangle\right)$. Note that

$$
c^{T} y-\nabla f(\bar{y})^{T} y=A \cdot\left(\frac{p(v, u)}{\bar{y}(u)}: u \in V\right)^{T} y \cdot(\tilde{g}-g(\bar{y})) \geq 0 \text { for all } y \in K_{\delta},
$$

while

$$
c^{T} \bar{y}-\nabla f(\bar{y})^{T} \bar{y}=A \cdot\left(\frac{p(v, u)}{\bar{y}(u)}: u \in V\right)^{T} \bar{y} \cdot(\tilde{g}-g(\bar{y})) \leq \delta^{\prime}
$$

It follows from (25), (26), and (27) that $c^{T} y+\delta^{\prime} \geq c^{T} \bar{y}$ for all $y \in K_{\delta}$.

Lemma 4 Given $t \in \mathbb{R}$ and $\delta \in(0,1)$ we can decide in time poly $\left(n, U, D^{k}, \log \frac{1}{\delta}\right)$ if the system (6)-(19) (resp., (13)-(16)) is infeasible, or find $y(v) \in\left[b^{-L}, b^{L}+\delta\right]$, for $v \in V^{\prime}$ and $y(v) \in\left[0, b^{L}+\delta\right]$, for $v \in V \backslash V^{\prime}$, such that the left hand sides of (6) -(8) (resp., (13))-(15)) are at least $b^{t} y(v)-\delta$ (resp., $\left.b^{-t} y(v)-\delta\right)$, for all $v \in V$.

Proof Let us consider the equivalent system (6) -(9); the proof for (21)-(24) is similar. Given a polynomial-time algorithm for the separation problem for the convex set $K_{\delta}$, a circumscribing ball of radius $H$ for $K_{\delta}$, and any $\epsilon^{\prime}>0$, the ellipsoid method terminates in $N:=O\left(n \log \frac{1}{\epsilon^{\prime}}+n^{2}|\log H|\right)$ calls to the separation algorithm using $\delta^{\prime}=2^{-O(N)}$, and either (i) finds a vector $y \in K_{\delta}$, or (ii) asserts that $\operatorname{vol}\left(K_{\delta}\right) \leq \epsilon^{\prime}$; see, e.g., Theorem 3.2.1 in [GLS88. In the first case, we get a vector $y$ satisfying the conditions in the statement of the lemma. In the second case, we conclude that $K_{\delta}$ and hence $K$ is empty if $\epsilon^{\prime}<\left(b^{-t} \delta\right)^{n}$. Indeed by Claim 1, if $K \neq \emptyset$ and $\epsilon:=b^{-t} \delta$, then $\operatorname{vol}\left(K_{\delta}\right) \geq \epsilon^{n}>\epsilon^{\prime}$, given a contradiction to the assertion in (ii).

By (20), the radius of the bounding ball can be chosen as $H:=2 b^{L}$. Furthermore, the ellipsoid method works only with numbers having precision of $O(N)$ bits. By Claim 2 , the separation problem can be solved in time $\operatorname{poly}\left(n, U, D^{k}, \log \frac{1}{\delta}\right)$.

Remark 1 By raising to inequalities (8) and (15) to power D, we obtain systems of polynomial inequalities. Khachiyan [Kha83, Kha84] gave a polynomial-time algorithm for (approximately) solving a system of convex polynomial inequalities. However, it is not possible to use this algorithm directly to solve the convex programs (6)-(9) and (13)-(16), since the polynomials obtained after raising inequalities (8) and (15) to power $D$ are not necessarily convex. For instance, the function $\sqrt{x y}-z$ is concave for $x, y, z \in \mathbb{R}_{+}$, while the function $x y-z^{2}$ is not.

\footnotetext{
${ }^{2}$ in case of $K_{\delta}^{\prime}$, they are rational numbers of denominator at most $n^{\Lambda D^{2} U}$

${ }^{3}$ For this part of the proof we can consider the restriction of $y$ to the set of positions reachable from $v$ by one move. We can also replace parallel edges by one edge; if $v$ is a position of chance then the transition probability of this edge is the sum of the transition probabilities of all corresponding parallel edges.
} 


\section{A Pseudo-polynomial algorithm for $k=O(1)$}

Let $\mathcal{G}$ be a BWR-game. Let $t_{\max }:=\max _{v \in V} \mu_{\mathcal{G}}(v)$ and $t_{\min }:=\min _{v \in V} \mu_{\mathcal{G}}(v)$. Define the top and bottom classes of $\mathcal{G}$ as $\mathcal{T}:=\left\{v \in V \mid \mu_{\mathcal{G}}(v)=t_{\max }\right\}$ and $\mathcal{B}:=\left\{v \in V \mid \mu_{\mathcal{G}}(v)=t_{\min }\right\}$, respectively.

Proposition 1 Top and bottom classes necessarily satisfy the following properties.

(i) There exists no arc $(v, u) \in E$ such that $v \in\left(V_{W} \cup V_{R}\right) \cap \mathcal{B}, u \notin \mathcal{B}$;

(ii) there exists no arc $(v, u) \in E$ such that $v \in\left(V_{B} \cup V_{R}\right) \cap \mathcal{T}, u \notin \mathcal{T}$;

(iii) there exists no arc $(v, u) \in E$ such that $v \in V_{W} \backslash \mathcal{T}, u \in \mathcal{T}$;

(iv) there exists no arc $(v, u) \in E$ such that $v \in V_{B} \backslash \mathcal{B}, u \in \mathcal{B}$;

(v) for every $v \in V_{W} \cap \mathcal{T}$, there exists an arc $(v, u) \in E$ such that $u \in \mathcal{T}$;

(vi) for every $v \in V_{B} \cap \mathcal{B}$, there exists an arc $(v, u) \in E$ such that $u \in \mathcal{B}$;

(vii) for every $v \in\left(V_{B} \cup V_{R}\right) \backslash \mathcal{T}$, there exists an arc $(v, u) \in E$ such that $u \notin \mathcal{T}$;

(viii) for every $v \in\left(V_{W} \cup V_{R}\right) \backslash \mathcal{B}$, there exists an arc $(v, u) \in E$ such that $u \notin \mathcal{B}$.

Proof All claims follow from the existence of a canonical form for $\mathcal{G}$, by Theorem 2 . Indeed, the existence of arcs forbidden by (i), (ii), (iii) and (iv), or the non-existence of arcs required by (v), (vi), (vii) and (viii) would violate the value equations (C1) of the canonical form.

Lemma 5 Consider the convex program defined by (6)-(8) (resp., [13)-(15)). Then for $t:=t_{\max }$ (resp., $\left.t:=t_{\mathrm{min}}\right)$, there is a feasible solution with $y(v) \geq b^{-L}$ for all $v \in \mathcal{T}$ (resp., $\left.v \in \mathcal{B}\right)$.

Proof Consider the game $\mathcal{G}[\mathcal{T}]$ (resp., $\mathcal{G}[\mathcal{B}]$ ) induced by the top class $\mathcal{T}$ (resp., the bottom class $\mathcal{B}$ ). Let $x \in \mathbb{R}^{\mathcal{T}}$ (resp., $x \in \mathbb{R}^{\mathcal{B}}$ ) be the potential vector guaranteed by Theorem 2 for the game $\mathcal{G}[\mathcal{T}]$ (resp., $\mathcal{G}[\mathcal{B}])$. Set $y(v):=b^{-x(v)}$ for $v \in \mathcal{T}\left(\right.$ resp., $y(v):=b^{x(v)}$ for $v \in \mathcal{B}$ ) and $y(v)=0$ for $v \in V \backslash \mathcal{T}$ (resp., $v \in V \backslash \mathcal{B}$ ). Then $y(v) \geq b^{-L}$ for all $v \in \mathcal{T}$ (resp., $v \in \mathcal{B}$ ). It is easy to verify by Proposition 1 that the system is feasible. Indeed, (6) is satisfied for every position $v \in V_{W} \cap \mathcal{T}$ (resp., $v \in V_{B} \cap \mathcal{B}$ ) by the definition of $x$ and Fact 1 ;

$$
\begin{gathered}
t_{\max } \leq \max _{u \mid(v, u) \in E}(r(v, u)+x(v)-x(u)) \leq \log _{b} \sum_{u \mid(v, u) \in E} b^{r(v, u)+x(v)-x(u)} \\
\text { (resp., } \left.-t_{\min } \leq-\min _{u \mid(v, u) \in E}(r(v, u)+x(v)-x(u)) \leq-\log _{b} \sum_{u \mid(v, u) \in E} b^{-r(v, u)-x(v)+x(u)}\right) .
\end{gathered}
$$

Moreover, for $v \in\left(V_{B} \cup V_{R}\right) \cap \mathcal{T}$ (resp., $v \in\left(V_{W} \cup V_{R}\right) \cap \mathcal{B}$ ) we have (7) and (8) (resp., (14) and (15)) satisfied by the definition of $x$ and Proposition 1 (ii) (resp., Proposition 1 1 (i)), while for $v \in V \backslash \mathcal{T}$ (resp., $v \in V \backslash \mathcal{B})$ (6)-(8) (resp., (13)-(15)) are trivially satisfied.

In the following we set $\delta(t):=\frac{1}{2} b^{-t-L}\left(1-\frac{1}{n}\right)$, where $\varepsilon:=\frac{1}{\sqrt{k} 2^{k / 2+1} D^{k+1}}$. Note that $b:=n^{\frac{2}{\varepsilon}}$.

Lemma 6 The values $t_{\max }$ and $t_{\min }$ can be found in time $\operatorname{poly}\left(n, U, D^{k}\right)$.

Proof We only show how to find $t_{\max }$; in a similar fashion we can determine $t_{\min }$. We apply Lemma 4 in a binary search manner to check the feasibility of the system (13)-(16) for $t \in[0, U]$ and $\delta(t)$ as specified above. Note that, by Lemma 1 $t_{\max } \in[0, U]$ can be written as a rational number with denominator at most $\frac{1}{2 \varepsilon}$. So we may restrict our search steps to integer multiples of $\frac{1}{2 \varepsilon}$. We stop the search when the length of the search interval becomes a constant multiple of $\frac{1}{2 \varepsilon}$, and then apply linear search for the remaining small interval. 
Suppose that the convex program (13)-(16) is infeasible. Then Theorem 2 implies that $t_{\max }>$ $t$. On the other hand, if $y \in \mathbb{R}^{V}$ is a $\delta(t)$-approximately feasible solution for (13)-(16), then as $\delta(t) \leq \frac{1}{2} b^{-L}$, the new vector $y^{\prime}:=2 y$ satisfies $y^{\prime}(v) \in\left[b^{-L}, 2 b^{L}+b^{-L}\right]$ for all $v \in V$. Also, $y^{\prime}$ satisfies (13)-(15) within an error of $2 \delta(t)$, that is, the left-hand sides of (13)-(15), when $y$ is replaced by $y^{\prime}$, are at least $b^{-t} y^{\prime}(v)-2 \delta(t)=b^{-t} y^{\prime}(v)-b^{-t-L}\left(1-\frac{1}{n}\right) \geq b^{-t-\log _{b} n} y^{\prime}(v)$. Set $x(v):=\log _{b} y^{\prime}(v)$. Then $x$ satisfies (10)-(12) with $t$ replaced by $t+\log _{b} n$. This in turn implies by Fact 1 that $M\left[r_{x}\right] \leq\left(t+2 \log _{b} n\right) \mathbf{1}=(t+\varepsilon) \mathbf{1}$. It follows then from Lemma 3 that $t_{\max } \leq t+\varepsilon$. Recall that we assume both $t$ and $t_{\max }$ are multiples of $2 \varepsilon$; hence, $t_{\max } \leq t$.

Since the number of binary search steps is at most $\log \frac{U}{2 \epsilon}=O(k \log (U D))$ and each step requires time $\operatorname{poly}\left(n, U, \log b, \log \frac{1}{\delta}\right)=\operatorname{poly}\left(n, U,{ }^{k}\right)$, the bound on the running time follows.

Lemma 7 We can find the top class $\mathcal{T}$ (resp., bottom class $\mathcal{B}$ ) in time $\operatorname{poly}\left(n, U, D^{k}\right)$.

Proof We can check if a vertex $w \in V$ belongs to the top class (resp., bottom class) as follows. We write the convex program (6)-(8) (resp., (13)-(15)) with $t:=t_{\max }$ (resp., $\left.t=t_{\min }\right)$ and with the additional constraint that $y(w) \geq b^{-L}$ and $y(v) \geq 0$ for all $v \in V \backslash\{w\}$. Then we check the feasibility of this system. If the system is infeasible then we know by Lemma 5 that $w \notin \mathcal{T}$ (resp., $w \notin \mathcal{B})$.

Suppose, on the other hand, that $y \in \mathbb{R}^{V}$ is a $\delta\left(t_{\max }\right)$-approximately (resp., $\delta\left(t_{\min }\right)$-approximately) feasible solution for (6)-(8) (resp., (13)-(15)). Then as in the proof of Lemma 6, the new vector $y^{\prime}:=2 y$ satisfies $y^{\prime}(w) \geq b^{-L}>0$, and the left-hand sides of (6)-(8) (resp., (13)-(15)), when $y$ is replaced by $y^{\prime}$, are at least $b^{t-\log _{b} n} y^{\prime}(w)$ (resp., $\left.b^{-t-\log _{b} n} y^{\prime}(w)\right)$.

Now we claim that $V^{+}:=\{v \in V: y(v)>0\} \subseteq \mathcal{T}$ (resp., $V^{+}:=\{v \in V: y(v)>0\} \subseteq \mathcal{B}$ ), which would in turn imply that $w \in \mathcal{T}$ (resp., $w \in \mathcal{B}$ ). Indeed, constraints (6)-(8) (resp., (13)-(15)), applied to $y$ replaced by $y^{\prime}$, imply that (i) if $v \in V_{W} \cap V^{+}$then there exists an arc $(v, u) \in E$ such that $u \in V^{+}$; (ii) if $v \in\left(V_{B} \cup V_{R}\right) \cap V^{+}$then all $\operatorname{arcs}(v, u) \in E$ must that $u \in V^{+}$(resp., (i) if $v \in V_{B} \cap V^{+}$then there exists an $\operatorname{arc}(v, u) \in E$ such that $u \in V^{+}$; (ii) if $v \in\left(V_{W} \cup V_{R}\right) \cap V^{+}$then all $\operatorname{arcs}(v, u) \in E$ must that $\left.u \in V^{+}\right)$. These imply that the game induced by $V^{+}$is well-defined and, by Lemma 2 (resp., Lemma 3), all its positions have value at least $t_{\max }$ (resp., at most $t_{\min }$ ). The lemma follows.

Finally, given the top and bottom classes, we can find an optimal pair of strategies in the games induced by $\mathcal{T}$ and $\mathcal{B}$, as stated in the next lemma.

Lemma 8 We can find optimal pairs of strategies in the games induced by the top class $\mathcal{T}$ and bottom class $\mathcal{B}$ in time $\operatorname{poly}\left(n, U, D^{k}\right)$.

Proof We prove the lemma only for $\mathcal{T}$; the proof for $\mathcal{B}$ can be done similarly. We solve two (feasible) systems, $\mathcal{S}_{1}$ defined by (지)-(9) on $\mathcal{G}[\mathcal{T}]$ and $\mathcal{S}_{2}$ defined by (13)-(15) on $\mathcal{G}[\mathcal{T}]$, with $t:=t_{\text {max }}$ to within an accuracy of $\delta\left(t_{\max }\right)$. Let $y^{1}, y^{2} \in \mathbb{R}^{\mathcal{T}}$ be the $\delta\left(t_{\max }\right)$-approximate solutions to $\mathcal{S}_{1}$ and $\mathcal{S}_{2}$, respectively. By the same arguments as in Lemma 6 the corresponding potential vectors $x^{1}, x^{2}$ (defined by $x^{1}(v):=-\log _{b}\left(2 y^{1}(v)\right)$ and $\left.x^{2}(v):=\log _{b}\left(2 y^{2}(v)\right)\right)$ ensure that $M\left[r_{x^{1}}\right] \geq\left(t_{\max }-\varepsilon\right) \mathbf{1}$ and $M\left[r_{x^{2}}\right] \leq\left(t_{\max }+\varepsilon\right) \mathbf{1}$. Since $\varepsilon$ is sufficiently small, by Lemmas 2] and 3, the locally optimal strategies defined by the operator $M$ with respect to $x^{1}$ and $x^{2}$ give optimal strategies for MAX and Min in $\mathcal{G}[\mathcal{T}]$, respectively.

Finally, we obtain Theorem 1 by combining the above lemmas with the algorithm in BEGM13b, BEGM15.

\section{References}

[AM09] D. Andersson and P. B. Miltersen. The complexity of solving stochastic games on graphs. In ISAAC, pages 112-121, 2009. 
[BEGM10] E. Boros, K. Elbassioni, V. Gurvich, and K. Makino. A pumping algorithm for ergodic stochastic mean payoff games with perfect information. In Proc. 14th IPCO, pages 341-354, 2010.

[BEGM13a] E. Boros, K. Elbassioni, V. Gurvich, and K. Makino. On canonical forms for zero-sum stochastic mean payoff games. Dynamic Games and Applications, 3(2):128-161, 2013.

[BEGM13b] Endre Boros, Khaled M. Elbassioni, Vladimir Gurvich, and Kazuhisa Makino. A pseudo-polynomial algorithm for mean payoff stochastic games with perfect information and a few random positions. In Automata, Languages, and Programming - 40th International Colloquium, ICALP 2013, Riga, Latvia, July 8-12, 2013, Proceedings, Part I, pages 220-231, 2013.

[BEGM15] E. Boros, K. Elbassioni, V. Gurvich, and K. Makino. A pseudo-polynomial algorithm for mean payoff stochastic games with perfect information and few random positions. CoRR, abs/1508.03431, 2015.

[BV01a] E. Beffara and S. Vorobyov. Adapting gurvich-karzanov-khachiyan's algorithm for parity games: Implementation and experimentation. Technical Report 2001020, Department of Information Technology, Uppsala University, available at: https://www.it.uu.se/research/reports/\#2001, 2001.

[BV01b] E. Beffara and S. Vorobyov. Is randomized gurvich-karzanov-khachiyan's algorithm for parity games polynomial? Technical Report 2001-025, Department of Information Technology, Uppsala University, available at: https://www.it.uu.se/research/reports/\#2001, 2001.

[BV05] H. Björklund and S. Vorobyov. Combinatorial structure and randomized subexponential algorithm for infinite games. Theoretical Computer Science, 349(3):347360,2005 .

[BV07] H. Björklund and S. Vorobyov. A combinatorial strongly sub-exponential strategy improvement algorithm for mean payoff games. Discrete Applied Mathematics, $155(2): 210-229,2007$.

[CH08] K. Chatterjee and T. A. Henzinger. Reduction of stochastic parity to stochastic meanpayoff games. Inf. Process. Lett., 106(1):1-7, 2008.

[CHKN14] K. Chatterjee, M. Henzinger, S. Krinninger, and D. Nanongkai. Polynomial-time algorithms for energy games with special weight structures. Algorithmica, 70(3):457-492, 2014.

[CJH04] K. Chatterjee, M. Jurdziński, and T. A. Henzinger. Quantitative stochastic parity games. In SODA '04, pages 121-130, Philadelphia, PA, USA, 2004. Society for Industrial and Applied Mathematics.

[EM79] A. Eherenfeucht and J. Mycielski. Positional strategies for mean payoff games. International Journal of Game Theory, 8:109-113, 1979.

[Gil57] D. Gillette. Stochastic games with zero stop probabilities. In A.W. Tucker M. Dresher and P. Wolfe, editors, Contribution to the Theory of Games III, in Annals of Mathematics Studies, volume 39, pages 179-187. Princeton University Press, 1957.

[GKK88] V. Gurvich, A. Karzanov, and L. Khachiyan. Cyclic games and an algorithm to find minimax cycle means in directed graphs. USSR Computational Mathematics and Mathematical Physics, 28:85-91, 1988.

[GLS88] M. Grötschel, L. Lovász, and A. Schrijver. Geometric Algorithms and Combinatorial Optimization. Springer, New York, 1988. 
[Hal07] N. Halman. Simple stochastic games, parity games, mean payoff games and discounted payoff games are all LP-type problems. Algorithmica, 49(1):37-50, 2007.

[HBV04] S. Sandberg H. Björklund and S. Vorobyov. A combinatorial strongly sub-exponential strategy improvement algorithm for mean payoff games. DIMACS Technical Report 2004-05, DIMACS, Rutgers University, 2004.

[JPZ06] M. Jurdziński, M. Paterson, and U. Zwick. A deterministic subexponential algorithm for solving parity games. In SODA '06, pages 117-123, New York, NY, USA, 2006. ACM.

[Jur98] M. Jurdziński. Deciding the winner in parity games is in UP $\cap$ co-UP. Inf. Process. Lett., 68(3):119-124, 1998.

[Jur00] M. Jurdziński. Games for Verification: Algorithmic Issues. PhD thesis, Department of Computer Science, University of Aarhus, Denmark, 2000.

[Kar78] R. M. Karp. A characterization of the minimum cycle mean in a digraph. Discrete Math., 23:309-311, 1978.

[Kha80] L. Khachiyan. Polynomial algorithms in linear programming. U.S.S.R. Comput. Math. Math. Phys., 20:53-72, 1980.

[Kha83] L. Khachiyan. Convexity and complexity in polynomial programming. In Proceedings of the International Congress of Mathematicians, Warsaw, page 15691577, 1983.

[Kha84] L. Khachiyan. Complexity of convex problems of real and integer programming. PhD thesis, The second thesis, manuscript, Computer Center of the USSR Academy of Sciences (in Russian), Moscow, 1984.

[KL93] A. V. Karzanov and V. N. Lebedev. Cyclical games with prohibition. Mathematical Programming, 60:277-293, 1993.

[Lit96] M. L. Littman. Algorithm for sequential decision making, CS-96-09. PhD thesis, Dept. of Computer Science, Brown Univ., USA, 1996.

[LL69] T. M. Liggett and S. A. Lippman. Stochastic games with perfect information and time-average payoff. SIAM Review, 4:604-607, 1969.

[MO70] H. Mine and S. Osaki. Markovian decision process. American Elsevier Publishing Co., New York, 1970.

[Mou76a] H. Moulin. Extension of two person zero sum games. Journal of Mathematical Analysis and Application, 5(2):490-507, 1976.

[Mou76b] H. Moulin. Prolongement des jeux à deux joueurs de somme nulle. Bull. Soc. Math. France, Memoire, 45, 1976.

[Pis99] N. N. Pisaruk. Mean cost cyclical games. Mathematics of Operations Research, 24(4):817-828, 1999.

[RF91] T. E. S. Raghavan and J. A. Filar. Algorithms for stochastic games: A survey. Mathematical Methods of Operations Research, 35(6):437-472, 1991.

[Sch09] Sven Schewe. From parity and payoff games to linear programming. In Mathematical Foundations of Computer Science 2009, 34th International Symposium, MFCS 2009, Novy Smokovec, High Tatras, Slovakia, August 24-28, 2009. Proceedings, pages 675686, 2009.

[Vor08] S. Vorobyov. Cyclic games and linear programming. Discrete Applied Mathematics, Special volume in Memory of Leonid Khachiyan (1952 - 2005), 156(11):2195-2231, 2008. 
[ZP96] U. Zwick and M. Paterson. The complexity of mean payoff games on graphs. Theoretical Computer Science, 158(1-2):343-359, 1996. 